\title{
Traumatic cataract: what, when, how
}

\author{
Ferenc Kuhn
}

Received: 20 March 2010 / Accepted: 2 April 2010 /Published online: 29 April 2010

(C) Springer-Verlag 2010

In the current issue of Graefe's Archive (see page ...), Drs. Rumelt and Rehany discuss some very important questions related to the timing of the removal of a traumatic cataract and implantation of an intraocular lens (IOL); they also touch upon the technique of cataract removal. According to the database of the world's largest collection of serious eye injuries with over 17,000 cases (United States Eye Injury Registry [USEIR], the surveillance arm of the American Society of Ocular Trauma), almost half of serious injuries involve lens damage [1]. The issue of how best to manage the patient with such trauma is therefore not a matter of concern for the individual only, but one with significant implications for society. The authors thus deserve a lot of credit for calling our attention to the topic, which otherwise receives surprisingly little attention in the literature.

The first thing we have to emphasize is that the gravest mistake an ophthalmologist can make is to treat an eye with a traumatic cataract as if it were an "ordinary" cataract, i.e., an age-related lens opacity. The injury is rarely limited to the lens alone; indeed, according to unpublished data from the USEIR, $48 \%$ of eyes with a traumatic cataract sustain injury to the posterior segment. Obviously, this is important since the visual outcome is determined mostly by the condition of the retina, much less by the consequences of the lens trauma. The two most notable exceptions to this rule are amblyopia, and whether the lens injury was treated properly; in other words, did the treatment cause any

\section{F. Kuhn $(\bowtie)$}

Helen Keller Foundation,

120111 th Ave S. Suite 300,

Birmingham, AL 35205, USA

e-mail: fkuhn@mindspring.com additional, easy-to-avoid-with-proper-technique complication. This is the basis upon which this editorial analyzes the paper by Rumelt and Rehany.

When the ophthalmologist encounters a traumatic cataract in the context of an open globe injury, the first thing to decide is whether to remove it primarily or defer it to be done as a secondary procedure, and this is where the first major difference compared to the age-related cataract environment arises.

No ophthalmologist has any difficulty establishing the diagnosis of cataract if it is age-related; however, in an eye with open globe injury, this often represents a significant challenge. The cornea may be cloudy; the anterior chamber filled with blood, fibrin, debris; the pupil may be small and difficult-to-dilate; or all three of these conditions may coexist. It is not that uncommon that a false-positive or false-negative diagnosis is made: presence of a traumatic cataract is assumed when none has occurred or the lens is deemed clear when in fact it is opacified. The ophthalmologist therefore better be absolutely sure that the lens is indeed irreversibly injured before committing to its removal; this is especially important since even if an intralenticular foreign body is present, the lens may remain clear (the cataract stationary) for extended periods of time [2].

Assuming a traumatic cataract is indeed present, the ophthalmologist's next decision is, as mentioned before, whether to remove it as a primary or a secondary procedure. Arguments can be made for both options; let's look at some of the most important factors based on which this decision can (should) be made.

Removing the lens primarily has definite advantages. It prevents complications such as intraocular pressure elevation from a swelling lens, a not-so-infrequent complication in younger people, and may occur rapidly; inflammation due to the release of antigenic lens proteins; allows, in the 
presence of a clear vitreous cavity, direct visualization of the retina in the postoperative period; and allows simultaneous implantation of an IOL, which is crucially important in patients in the amblyopic age (see below). Primary lens removal is especially beneficial if there is vitreous hemorrhage and severe lens damage; the "vitreous-lens admixture" is a potent inciter of proliferative vitreoretinopathy (PVR) [3]. Another benefit of primary lens removal is sparing the patient the inconvenience (and cost) of an additional procedure.

Conversely, secondary lens removal has its own distinct advantages: the eye may be more quiet (should the ophthalmologist have been applying judicious topical corticosteroid therapy); the diagnosis no more uncertain; the removal time and method optimally been chosen; and the decision regarding the timing of IOL implantation determined.

If one reviews all the advantages on both sides of the argument, it is obvious that there is no clear-cut answer, and indeed this is what Rumelt and Rehany found in their study. Nevertheless, the author of this editorial would like to add his own take on the issue, further elaborating on what the authors discuss: the decision is, and should be, influenced not only by the ophthalmologist's personal preference but also by the infrastructure of the facility where the primary surgery is done. This is very important, because no ophthalmologist should be in a position, no eye/patient should be subjected to the consequences of a halfcompleted surgery or one that is compromised because of the lack of equipment or material. If the facility is not equipped with providing after-hours the surgeon with the full array of equipment, instruments, and material, and a full and knowledgeable staff, it is better not to contemplate primary lens removal. Unfortunately, in the age of university hospitals closing their surgical doors at 5:00 pm in the afternoon, this is becoming an ever-increasing issue, and not taking it into consideration is a major cause of suboptimal intervention.

The next issue to be discussed here is the method of lens removal, be it primary or secondary. The authors employed three techniques in their case series: phacoemulsification, and intra- and extracapsular extraction (ICCE and ECCE). This deserves further analysis because, as mentioned before, the surgeon must avoid iatrogenic damage: inflicting tissue damage intraoperatively by using an inappropriate technique or employing the proper technique erroneously.

How should one make the choice regarding the removal technique of a traumatic cataract? There are two major issues to consider: first, the age of the patient; second, the condition of the posterior capsule (we forgo discussing what to do in eyes with lens subluxation since this was properly addressed by the authors: they used ICCE instead of trying to implant a capsular tension ring, for which again they deserve a lot of credit).
Why is the age of the patient important? Because the younger the patient, the more pronounced the connection between the posterior capsule and the anterior vitreous centrally; and the central vitreous is anatomically connected to the peripheral retina at the vitreous base. What this means is that traction on the anterior vitreous face is transmitted to the retina, and the younger the patient, the greater the risk. The average age in Rumelt and Rehany's study was 25 years, young enough to make this a real danger.

Phacoemulsification and ECCE represent the same principle: aspiration without cutting. This method is, of course, fine - unless and until vitreous is encountered. Once the handpiece aspirates vitreous, traction on the retinal periphery is unavoidable, and it is beyond the surgeon's control whether a retinal break ensues. Obviously, the postoperative identification of such a retinal break can be explained by the surgeon: "this is a preexisting break", and assuming no scleral indentation was performed during lens removal surgery, this explanation can never be disproved.

There are two scenarios of aspirating vitreous during lens removal: vitreous that has prolapsed through/around the zonules (lens sub/luxation), which is relatively easy to determine, especially if the surgeon uses triamcinolone acetonide crystals to delineate the vitreous. However, the second scenario does represent a real problem: identifying vitreous that has prolapsed, prior to or during, phacoemulsification/ECCE, into the lens itself.

To make sure that no such vitreous prolapse has occurred, it is vital to know the condition of the posterior capsule. Mester and Kuhn in their unpublished study did just that; they found that in the Hungarian Eye Injury Registry, $23 \%$ of eyes with lens injury had been assumed to have a posterior capsule lesion and thus an increased risk of vitreous prolapse into the lens. Intraoperatively, the rate of vitreous prolapse was $45 \%$; in other words, in half of eyes with traumatic cataract, the surgeon should expect to encounter vitreous while aspirating lens material.

In certain cases, obviously, the surgeon can be sure that no such vitreous prolapse has occurred; in other cases, he knows it has (e.g., a posteriorly located intraocular foreign body that entered the vitreous cavity through the lens). In the former cases, phacoemulsification (ECCE) is the ideal lens-removal technique; but in the latter cases, phacoemulsification is like playing Russian roulette. If the bullet was in the chamber, it is likely that a retinal break/detachment will be found postoperatively.

For his reason, it is rather surprising that the authors did not utilize pars plana (or limbal) lensectomy in any of their cases. The obvious advantage of using the vitrectomy probe for cataract extraction is that whether or not vitreous is encountered, no traction is exerted at the vitreous base, eliminating the risk of an iatrogenic retinal break. In the 
authors' series, $15 \%$ of eyes developed a retinal tear, and of course we will never know how many of these may been avoided by opting for lensectomy instead of phacoemulsification/ECCE.

The resistance to employing lensectomy originates in the fact that it is almost always impossible to retain the posterior capsule with this technique; rather, it is removed and the anterior capsule is typically left behind (in eyes with high risk of PVR both capsules should be removed, but discussing this is beyond the scope of this editorial). The lack of a posterior capsule means that the IOL will be "out-of-the-bag": it will be placed in the sulcus (with sutures if necessary), fixed to the iris, or in the anterior chamber. Either of these solutions is less ideal than the inthe-bag option; however, either is preferable to causing a retinal break by forgoing the one safe technique: lensectomy. It is a much lesser evil to "unnecessarily" remove the posterior capsule than to creating an iatrogenic break-even if the surgeon convinces himself that the retinal break discovered postoperatively must have been preexisting. It must also be added that the posterior capsule has a tendency to shrink in these severely injured eyes, exerting traction on the ciliary body, and be responsible for phthisis (see above: removal of both capsules should be considered if the risk of capsule-shrinkage is great).

Finally, the issue of IOL implantation: should it be concurrent with cataract removal or be done as a secondary procedure?

Again, there are strong arguments on both sides. In the amblyopic-age child, the sooner the lens is removed and IOL implanted, as correctly pointed out by Rumelt and Rehany, the better; even then, it will be a struggle to maintain vision in the injured eye.

Primary IOL implantation has additional benefits. There is no synechia formation that threatens "closing" the bag by the time secondary implantation can be carried out; it is more convenient to the patient, and it is less expensive.

On the other hand, it may be difficult if not impossible to determine the correct IOL power prior to surgery (often the other eye serves as a guide); the inflammatory debris can settle on the IOL surface, and it may require postoperative YAG laser or even surgery to cleanse it; and last but not least, the edge of the IOL will interfere with the surgeon's visualization of the peripheral retina should subsequent PVR development necessitate vitrectomy. This author's general rule is that primary IOL implantation is preferred, unless the retinal damage is extensive or the PVR risk high.

In summary, the vast majority of ophthalmologists who encounter a traumatic cataract have sufficient experience in lens extraction (and IOL implantation) in a non-traumatic setting. What every ophthalmologist must accept is that an injured lens requires many individualized, consciously made decisions regarding what to do when and how to achieve the best possible outcome. Trying to adapt the eye to the situation, rather than adapting the surgical procedure to the actual situation, is prone to lead to further, avoidable complications.

\section{References}

1. May D, Kuhn F, Morris R, Witherspoon D, Danis R, Matthews P, Mann L (2000) The epidemiology of serious eye injuries from the United States Eye Injury Registry. Graefe's Archives for Clinical and Experimental Ophthalmology 238:153-157

2. Pieramici D, Capone AJ, Rubsamen P, Roseman R (1996) Lens preservation after intraocular foreign body injuries. Ophthalmology 103:1563-1567

3. Cardillo J, Stout J, LaBree L, Azen S, Omphroy L, Cui J, Kimura H, Hinton D, Ryan S (1997) Posttraumatic proliferative vitreoretinopathy. The epidemiologic profile, onset, risk factors, and visual outcome. Ophthalmology 104:1166-1173 